\title{
Antiretroviral Agents and Prevention of Malaria in HIV-Infected Ugandan Children
}

\author{
Jane Achan, M.Med., Abel Kakuru, M.D., Gloria Ikilezi, M.D., \\ Theodore Ruel, M.D., Tamara D. Clark, M.P.H., Christian Nsanzabana, Ph.D., \\ Edwin Charlebois, M.P.H., Ph.D., Francesca Aweeka, Pharm.D., \\ Grant Dorsey, M.D., Ph.D., Philip J. Rosenthal, M.D., Diane Havlir, M.D., \\ and Moses R. Kamya, M.Med., Ph.D.
}

From the Departments of Pediatrics and Child Health (J.A.) and Medicine (M.R.K.), Makerere University College of Health Sciences, and the Infectious Diseases Research Collaboration (J.A., A.K., G.I., M.R.K.) - both in Kampala, Uganda; and the Departments of Pediatrics (T.R.), Medicine (T.D.C., C.N., E.C., G.D., P.J.R., D.H.), and Clinical Pharmacy, Drug Research Unit (F.A.), University of California, San Francisco, San Francisco. Address reprint requests to Dr. Achan at Makerere University College of Health Sciences, P.O. Box 7475 , Kampala, Uganda, or at achanj@yahoo.co.uk.

N EnglJ Med 2012;367:2110-8. DOI: 10.1056/NEJMoal200501

Copyright @ 2012 Massachusetts Medical Society.
A BSTRACT

BACKGROUND

Human immunodeficiency virus (HIV) protease inhibitors show activity against Plasmodium falciparum in vitro. We hypothesized that the incidence of malaria in HIVinfected children would be lower among children receiving lopinavir-ritonavirbased antiretroviral therapy (ART) than among those receiving nonnucleoside reverse-transcriptase inhibitor (NNRTI)-based ART.

METHODS

We conducted an open-label trial in which HIV-infected children 2 months to 5 years of age who were eligible for ART or were currently receiving NNRTI-based ART were randomly assigned to either lopinavir-ritonavir-based ART or NNRTI-based ART and were followed for 6 months to 2 years. Cases of uncomplicated malaria were treated with artemether-lumefantrine. The primary end point was the incidence of malaria.

RESULTS

We enrolled 176 children, of whom 170 received the study regimen: 86 received NNRTI-based ART, and 84 lopinavir-ritonavir-based ART. The incidence of malaria was lower among children receiving the lopinavir-ritonavir-based regimen than among those receiving the NNRTI-based regimen (1.32 vs. 2.25 episodes per person-year; incidence-rate ratio, 0.59 ; $95 \%$ confidence interval [CI], 0.36 to 0.97 ; $\mathrm{P}=0.04$ ), as was the risk of a recurrence of malaria after treatment with artemether-lumefantrine ( $28.1 \%$ vs. $54.2 \%$; hazard ratio, $0.41 ; 95 \% \mathrm{CI}, 0.22$ to 0.76 ; $\mathrm{P}=0.004)$. The median lumefantrine level on day 7 after treatment for malaria was significantly higher in the lopinavir-ritonavir group than in the NNRTI group. In the lopinavir-ritonavir group, lumefantrine levels exceeding $300 \mathrm{ng}$ per milliliter on day 7 were associated with a reduction of more than $85 \%$ in the 63 -day risk of recurrent malaria. A greater number of serious adverse events occurred in the lopinavirritonavir group than in the NNRTI group ( $5.6 \%$ vs. $2.3 \%, \mathrm{P}=0.16$ ). Pruritus occurred significantly more frequently in the lopinavir-ritonavir group, and elevated alanine aminotransferase levels significantly more frequently in the NNRTI group.

\section{CONCLUSIONS}

Lopinavir-ritonavir-based ART as compared with NNRTI-based ART reduced the incidence of malaria by $41 \%$, with the lower incidence attributable largely to a significant reduction in the recurrence of malaria after treatment with artemetherlumefantrine. Lopinavir-ritonavir-based ART was accompanied by an increase in serious adverse events. (Funded by the Eunice Kennedy Shriver National Institute of Child Health and Human Development; ClinicalTrials.gov number, NCT00978068.) 
ALARIA AND HUMAN IMMUNODEFIciency virus (HIV) infection impose immense and overlapping burdens in sub-Saharan Africa. Prophylaxis with trimethoprim-sulfamethoxazole and insecticide-treated bed nets reduce the incidence of malaria among HIV-infected children, but protection is incomplete, and even with the use of these protective strategies, the burden of malaria remains great in areas of high-intensity transmission. ${ }^{1}$ Thus, new approaches to the prevention of malaria are an important public health priority.

Antiretroviral protease inhibitors show in vitro activity against Plasmodium falciparum ${ }^{2-4}$ (the cause of most malaria cases in Africa), probably owing to inhibition of plasmodial aspartic proteases that are biochemically similar to the HIV-1 protease. $^{3,5}$ Lopinavir is the most potent of these inhibitors and is active at levels well below those achieved with standard doses of coformulated lopinavir-ritonavir. ${ }^{2,3}$ Since lopinavir-ritonavir is increasingly available for the treatment of HIV infections in Africa, it may represent a valuable tool for the prevention of malaria. However, because ritonavir inhibits the metabolism of many drugs, it has the potential for interactions with antimalarial agents, including widely used artemisinin-based combination therapies, potentially affecting the efficacy and safety of the drugs. ${ }^{6}$ We assessed the incidence of malaria in HIV-infected Ugandan children who were randomly assigned to receive a lopinavir-ritonavirbased antiretroviral regimen or a nonnucleoside reverse-transcriptase inhibitor (NNRTI)-based antiretroviral regimen.

\section{METHODS}

\section{STUDY PARTICIPANTS}

We conducted the study in Tororo, Uganda, an area of high-intensity malaria transmission. ${ }^{7}$ Eligible children were 2 months to 5 years of age, with confirmed HIV infection. Participants had either never received antiretroviral therapy (ART) and were eligible for initiation of ART according to national guidelines or were currently receiving standard first-line ART, comprising one NNRTI plus two nucleoside reverse-transcriptase inhibitors (NRTIs), and had an HIV RNA level of less than 400 copies per milliliter, as assessed in the most recent measurement during the preceding 6 months. A complete description of the entry criteria is provided in the Supplementary Appen- dix and in the protocol, both of which are available with the full text of this article at NEJM.org. The parents or guardians provided written informed consent for the participation of their children in the trial.

\section{STUDY DESIGN}

This was an open-label trial with randomization stratified at the time of enrollment according to prior or no prior receipt of ART. Randomization was performed in permuted blocks of 2 or 4 . Participants received either lopinavir-ritonavir (Abbott Laboratories) plus two NRTIs or an NNRTI - nevirapine (for children $<3$ years of age) or efavirenz (for children $\geq 3$ years of age) - plus two NRTIs. The NRTIs that were used in the regimens were lamivudine and zidovudine, with stavudine or abacavir replacing zidovudine in children who had anemia. Children who were already receiving ART were randomly assigned to continue their current regimen or to switch to lopinavir-ritonavir while continuing the same NRTIs. Parents and guardians received counseling on adherence before the children underwent randomization and were given a 4-week supply of ART at each monthly visit. Insulated coolers were provided when liquid lopinavir-ritonavir was dispensed for young children. Adherence to ART was assessed monthly on the basis of caregiver reports and pill counts.

\section{STUDY PROCEDURES}

At the time of enrollment, children received a long-lasting insecticide-treated bed net, a hygienic water-storage container, multivitamins, and trimethoprim-sulfamethoxazole to be taken daily. Participants received all their medical care at a study clinic that was open every day. Routine visits were scheduled every 4 weeks, and routine laboratory tests were performed every 12 weeks. Parents or guardians were encouraged to bring a child to the clinic any time the child was ill. In the case of children who presented with a documented fever (a tympanic temperature $\geq 38.0^{\circ} \mathrm{C}$ ) or a history of fever in the previous 24 hours, blood was obtained by finger prick for examination of a thick blood smear. The diagnosis of malaria was made if the smear was positive for malaria parasites. Children with uncomplicated malaria were treated with artemether-lumefantrine, which is the recommended first-line treatment in Uganda, and the parents or guardians were instructed to bring the children back for 
follow-up evaluation on days $1,2,3,7,14,21$, and 28. The administration of each first daily dose of artemether-lumefantrine was directly observed in the study clinic, and each second daily dose was administered at home. A complete blood count and measurement of the alanine aminotransferase level were performed on days 0 and 28. From July 13, 2010, through June 8, 2011, blood samples were obtained by means of finger prick 7 days after initiation of artemether-lumefantrine therapy, for measurement of lumefantrine levels. After November 15, 2010, electrocardiograms were obtained and were read by trained study physicians on days 0 and 3 to assess corrected QT (QTc) intervals. Outcomes of malaria treatment were classified according to World Health Organization (WHO) guidelines. ${ }^{8}$ Adverse events were assessed at every visit and were graded according to standardized criteria. ${ }^{9}$

\section{STUDY OVERSIGHT}

The study was approved by the Makerere University School of Medicine Research and Ethics Committee, the Uganda National Council for Science and Technology, and the University of California, San Francisco, Committee for Human Research. Lopinavir-ritonavir was donated by Abbott Laboratories, which played no role in the design of the study, the accrual or analysis of the data, or the preparation of the manuscript. All other antiretroviral drugs were available through existing distribution systems of the Uganda Ministry of Health, through Tororo District Hospital; in the event that any of these drugs were not available through this process, the drugs were purchased by the study team from a reputable pharmacy. The antimalarial drugs were purchased by the study team. All the authors vouch for the completeness and accuracy of the data and analyses and for the fidelity of the study to the protocol.

\section{LABORATORY PROCEDURES}

Parasite density was assessed from blood smears as described previously..$^{10}$ If a participant had a recurrence of malaria 4 to 63 days after the initiation of malaria therapy, genotyping was performed, as described previously, to distinguish recrudescence from new infection. ${ }^{10}$ Lumefantrine levels were measured in $25-\mu \mathrm{l}$ samples as described previously. ${ }^{11}$ The interassay and intra- assay precision (percentage coefficient of variation) was 5.3 to $6.1 \%$ and 2.2 to $10 \%$, respectively. The interassay and intra-assay accuracy was 103.5 to $107.1 \%$ and 99.5 to $109.7 \%$, respectively. The lower limit of quantification was $50 \mathrm{ng}$ per milliliter. At the time of the diagnosis of malaria, in vitro culturing of selected P. falciparum isolates was performed as described previously. ${ }^{12}$ After 2 to 4 weeks, aliquots were frozen in glycerol and stored in liquid nitrogen; they were subsequently thawed and cloned by means of a limiting-dilution technique. ${ }^{13}$ Drug-interaction studies were performed on clones from two different patients with the use of the checkerboard technique, as described previously. ${ }^{2}$

\section{STUDY END POINTS}

The primary end point was the incidence of malaria, which was defined as the number of incident episodes of malaria per time at risk. Malaria that was diagnosed within 14 days after a prior episode was not considered to be an incident event. The time at risk was calculated as the time from the day after initiation of study treatment to the last day of observation, minus 14 days after each incident episode of malaria. In the calculation of the time at risk, discounting the 14 days after each incident episode of malaria is a standard approach. Secondary outcomes included the incidence of complicated malaria, the efficacy and safety of antimalarial therapy, and pharmacokinetic characteristics of lumefantrine.

\section{STATISTICAL ANALYSIS}

To test the hypothesis that lopinavir-ritonavirbased ART would reduce the incidence of malaria, we assumed that the incidence of malaria in the NNRTI group would be 0.70 episodes per person-year and estimated that we would need a sample of 300 participants for the study to have $80 \%$ power to show a $35 \%$ reduction in the incidence of malaria in the lopinavir-ritonavir group, at a two-sided significance level of 0.05 . We subsequently observed an incidence of malaria in the NNRTI group that was higher than anticipated (2.19 episodes per person-year) and revised the sample size to 150 participants, who would be followed for at least 6 months.

The statistical analysis was performed according to the intention-to-treat principle, with the use of Stata software, version 11. For the between- 


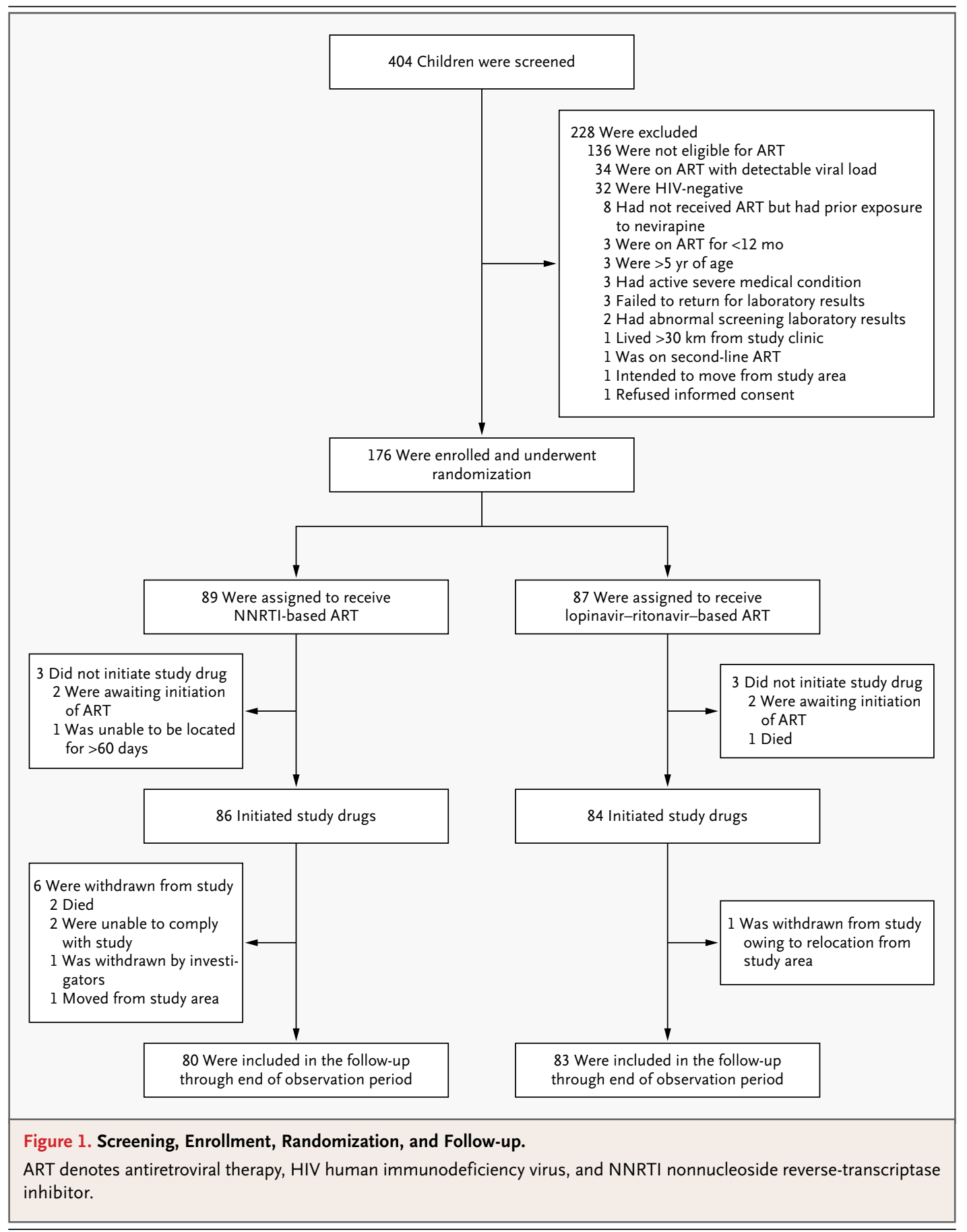

group comparisons of the incidence of malaria, we used a negative binomial regression model. Time to events was estimated with the use of the Kaplan-Meier product-limit formula, and comparisons were made with the use of a Cox proportional-hazards model with adjustment for repeated measures in the same patient. Pairwise comparisons of categorical and continuous variables at the level of each episode of malaria were made with the use of generalized estimating equations with adjustment for repeated measures in the same patient and with exchangeable correlation and robust 
standard errors. P values of less than 0.05 were considered to indicate statistical significance.

\section{RESULTS}

STUDY PARTICIPANTS AND FOLLOW-UP

From September 2009 through July 2011, a total of 404 children were screened for eligibility; 228 were found not to be eligible, including 136 who did not meet the criteria for initiation of ART (Fig. 1). A total of 176 children underwent ran-

\begin{tabular}{|c|c|c|}
\hline Characteristic & $\begin{array}{l}\text { NNRTI-Based } \\
\text { ART }(\mathrm{N}=86)\end{array}$ & $\begin{array}{l}\text { Lopinavir-Ritonavir- } \\
\text { Based ART }(\mathrm{N}=84)\end{array}$ \\
\hline \multicolumn{3}{|l|}{ Age $-\mathrm{yr}$} \\
\hline Median & 3.1 & 2.9 \\
\hline Range & $0.5-5.9$ & $0.7-6.0$ \\
\hline Female sex — no. (\%) & $41(48)$ & $41(49)$ \\
\hline No previous ART — no. (\%) & $58(67)$ & $57(68)$ \\
\hline \multicolumn{3}{|l|}{ WHO clinical HIV stage — no. (\%) } \\
\hline 1 & $66(77)$ & $60(71)$ \\
\hline II & $15(17)$ & $16(19)$ \\
\hline III & $1(1)$ & $2(2)$ \\
\hline IV & $4(5)$ & $6(7)$ \\
\hline \multicolumn{3}{|l|}{ CD4 percentage $\dagger$} \\
\hline \multicolumn{3}{|l|}{ No previous ART } \\
\hline Median & 16 & 14 \\
\hline Range & $2-43$ & $2-44$ \\
\hline \multicolumn{3}{|l|}{ Previous ART } \\
\hline Median & 30 & 31 \\
\hline Range & $10-45$ & $8-51$ \\
\hline \multicolumn{3}{|l|}{ Viral load - $\log _{10}$ copies/ml } \\
\hline \multicolumn{3}{|l|}{ No previous ART } \\
\hline Median & 5.5 & 5.4 \\
\hline Range & BLD-6.4 & BLD-6.4 \\
\hline \multicolumn{3}{|l|}{ Previous ART } \\
\hline Median & BLD & BLD \\
\hline Range & BLD & BLD \\
\hline Hemoglobin $-g / d l$ & $10.6 \pm 1.5$ & $10.4 \pm 1.3$ \\
\hline $\begin{array}{c}\text { Positive blood smear for asexual } \\
\text { parasites — no. (\%) }\end{array}$ & $11(13)$ & $10(12)$ \\
\hline
\end{tabular}

* Plus-minus values are means $\pm S D$. There were no significant differences between the groups in baseline characteristics. BLD denotes below the level of detection ( $<400$ copies per microliter), and WHO World Health Organization. $\uparrow \mathrm{CD} 4$ percentage is the preferred measurement for children younger than 5 years of age, as recommended by the WHO. The percentage represents the percentage of white cells that are CD4 cells. domization: 89 were assigned to NNRTI-based ART and 87 to lopinavir-ritonavir-based ART. Study drugs were initiated in 86 children in the NNRTI group and 84 in the lopinavir-ritonavir group, and these children were followed for a median of 366 days. The baseline characteristics were similar between the two groups (Table 1). During monthly routine assessments, $97 \%$ of caregivers reported $100 \%$ adherence to ART.

\section{INCIDENCE OF MALARIA}

Among the study participants, there were 285 new episodes of malaria during 162 person-years of follow-up; all the episodes of malaria were due to P. falciparum infection. The incidence of malaria was significantly lower in the lopinavirritonavir group than in the NNRTI group (1.32 vs. 2.25 episodes per person-year), resulting in protective efficacy of $41 \%$ with lopinavir-ritonavir (incidence rate ratio, 0.59; 95\% confidence interval [CI], 0.36 to $0.97 ; \mathrm{P}=0.04$ ) (Table 2). Complicated malaria occurred infrequently, with a similar incidence in the two groups. To assess the effect of ART independently of potential interactions with antimalarial therapy after treatment for malaria, we compared the two groups with respect to the time to the first episode of malaria (Fig. 2A). The 6-month risk of a first episode of malaria was $40.7 \%$ in the lopinavir-ritonavir group, as compared with $52.5 \%$ in the NNRTI group (hazard ratio with lopinavir-ritonavir, $0.71 ; 95 \% \mathrm{CI}, 0.45$ to $1.12 ; \mathrm{P}=0.14$ ) (Table 2).

\section{EFFICACY AND SAFETY OUTCOMES AFTER ANTIMALARIAL THERAPY}

Of the 285 new episodes of malaria, 281 (98.6\%) were uncomplicated and were treated with artemether-lumefantrine. To assess the effect of potential interactions between ART and artemether-lumefantrine, the risks of recurrent parasitemia and recurrent malaria were compared between the two groups. The 28-day risk of recurrent parasitemia was significantly lower in the lopinavir-ritonavir group than in the NNRTI group $(14.0 \%$ vs. $40.8 \%, \mathrm{P}=0.004)$, as was the 63-day risk of recurrent malaria ( $28.1 \%$ vs. $54.2 \%$, $\mathrm{P}=0.004$ ) (Table 2 and Fig. 2B). Among patients in whom parasites were cleared by 7 days, there were 111 episodes of recurrent malaria within 63 days; 107 cases were successfully genotyped, and all were classified as new infections. Resolution of fever, parasite clearance, and recovery of mean 


\begin{tabular}{|c|c|c|c|c|c|c|c|c|}
\hline \multirow[t]{2}{*}{ Outcome } & \multicolumn{3}{|c|}{ NNRTI-Based ART } & \multicolumn{3}{|c|}{ Lopinavir-Ritonavir-Based ART } & \multirow[t]{2}{*}{$\begin{array}{c}\text { Incidence-Rate Ratio } \\
(95 \% \mathrm{Cl})\end{array}$} & \multirow[t]{2}{*}{ P Value } \\
\hline & $\begin{array}{l}\text { No. of } \\
\text { Events }\end{array}$ & $\begin{array}{l}\text { Person-Yr } \\
\text { at Risk }\end{array}$ & $\begin{array}{l}\text { Episodes/ } \\
\text { Person-Yr }\end{array}$ & $\begin{array}{l}\text { No. of } \\
\text { Events }\end{array}$ & $\begin{array}{l}\text { Person-Yr } \\
\text { at Risk }\end{array}$ & $\begin{array}{l}\text { Episodes/ } \\
\text { Person-Yr }\end{array}$ & & \\
\hline \multicolumn{9}{|l|}{ Episodes of malaria } \\
\hline All episodes & 176 & 78.2 & 2.25 & 109 & 82.3 & 1.32 & $0.59(0.36-0.97)$ & 0.04 \\
\hline \multirow[t]{4}{*}{ Complicated malaria } & 2 & 78.2 & 0.026 & 2 & 82.3 & 0.024 & $0.80(0.06-11.16)$ & 0.87 \\
\hline & \multicolumn{3}{|c|}{ NNRTI-Based ART } & \multicolumn{3}{|c|}{ Lopinavir-Ritonavir-Based ART } & $\begin{array}{l}\text { Hazard Ratio with } \\
\text { Lopinavir-Ritonavir } \\
(95 \% \mathrm{Cl})\end{array}$ & P Value \\
\hline & $\begin{array}{l}\text { No. of } \\
\text { Events }\end{array}$ & Cumulat & tive Risk & $\begin{array}{l}\text { No. of } \\
\text { Events }\end{array}$ & Cumula & tive Risk & & \\
\hline & \multicolumn{3}{|c|}{$\%(95 \% \mathrm{Cl})$} & \multicolumn{3}{|c|}{$\%(95 \% \mathrm{Cl})$} & & \\
\hline \multicolumn{9}{|l|}{ Risk of malaria } \\
\hline $\begin{array}{l}\text { 6-mo risk of first episode } \\
\text { of malaria* }\end{array}$ & 42 & \multicolumn{2}{|c|}{$52.5(42.0-63.9)$} & 33 & \multicolumn{2}{|c|}{$40.7(30.9-52.2)$} & $0.71(0.45-1.12)$ & 0.14 \\
\hline $\begin{array}{l}\text { 28-day risk of recurrent } \\
\text { parasitemia } \dagger\end{array}$ & 174 & \multicolumn{2}{|c|}{$40.8(33.9-48.6)$} & 107 & \multicolumn{2}{|c|}{$14.0(8.7-22.2)$} & $0.31(0.14-0.68)$ & 0.004 \\
\hline $\begin{array}{l}\text { 63-day risk of recurrent } \\
\text { malaria } \dagger\end{array}$ & 174 & \multicolumn{2}{|c|}{$54.2(46.4-62.2)$} & 107 & \multicolumn{2}{|c|}{$28.1(20.2-38.3)$} & $0.41(0.22-0.76)$ & 0.004 \\
\hline
\end{tabular}

* The 6-month risk of a first episode of malaria was assessed in 86 patients in the NNRTI group and 84 in the lopinavir-ritonavir group. Among patients who were followed for 6 months, malaria did not develop in 34 patients in the NNRTI group and 44 in the lopinavir-ritonavir group; data on 10 patients in the NNRTI group and 7 in the lopinavir-ritonavir group were censored before the 6-month follow-up assessment.

$\dagger$ The risk of recurrence was assessed among patients who had had uncomplicated malaria that had been treated with artemether-lumefantrine.

hemoglobin levels after antimalarial treatment were similar in the two groups (Table S1 in the Supplementary Appendix). There were no significant differences in the rates of adverse events over the course of the 28-day period after antimalarial therapy, except that pruritus was more common in the lopinavir-ritonavir group $(5.6 \%$ vs. $1.2 \%, P=0.04$ ), and elevation of alanine aminotransferase levels occurred more frequently in the NNRTI group $(13.5 \%$ vs. $3.3 \%, \mathrm{P}=0.003)(\mathrm{Ta}-$ ble S2 in the Supplementary Appendix). A total of 10 serious adverse events occurred during the standard WHO 28-day period for malaria followup, with a trend toward a higher frequency in the lopinavir-ritonavir group ( $5.6 \%$ vs. $2.3 \%, \mathrm{P}=0.16$ ). All the serious adverse events were considered to be unrelated to the study drugs, with the exception of one episode of the Stevens-Johnson syndrome in the NNRTI group, which led to discontinuation of the study drug, and two episodes of neutropenia in the lopinavir-ritonavir group, which resolved without discontinuation of therapy. Electrocardiograms obtained on day 3 after initiation of treatment for malaria in a subgroup of 120 patients showed no episodes of prolongation of the QTc interval (Table S2 in the Supplementary Appendix).

\section{PHARMACOKINETIC CHARACTERISTICS OF MALARIA THERAPY AND DRUG INTERACTIONS}

During the period in which blood samples were obtained to assess drug levels, there were 103 episodes of malaria in the NNRTI group and 73 in the lopinavir-ritonavir group; lumefantrine levels were successfully measured on day 7 after initiation of malaria treatment in the case of 92 episodes $(89 \%)$ and 65 episodes (89\%), respectively. The median lumefantrine level was significantly higher in the lopinavir-ritonavir group than in the NNRTI group (926 ng per milliliter [interquartile range, 473 to 1910] vs. 200 ng per milliliter [interquartile range, 108 to 510], $\mathrm{P}<0.001$ ). In addition, in the NNRTI group, the median lumefantrine level on day 7 was significantly higher among the 67 patients who were taking nevirapine than among the 25 patients who were 

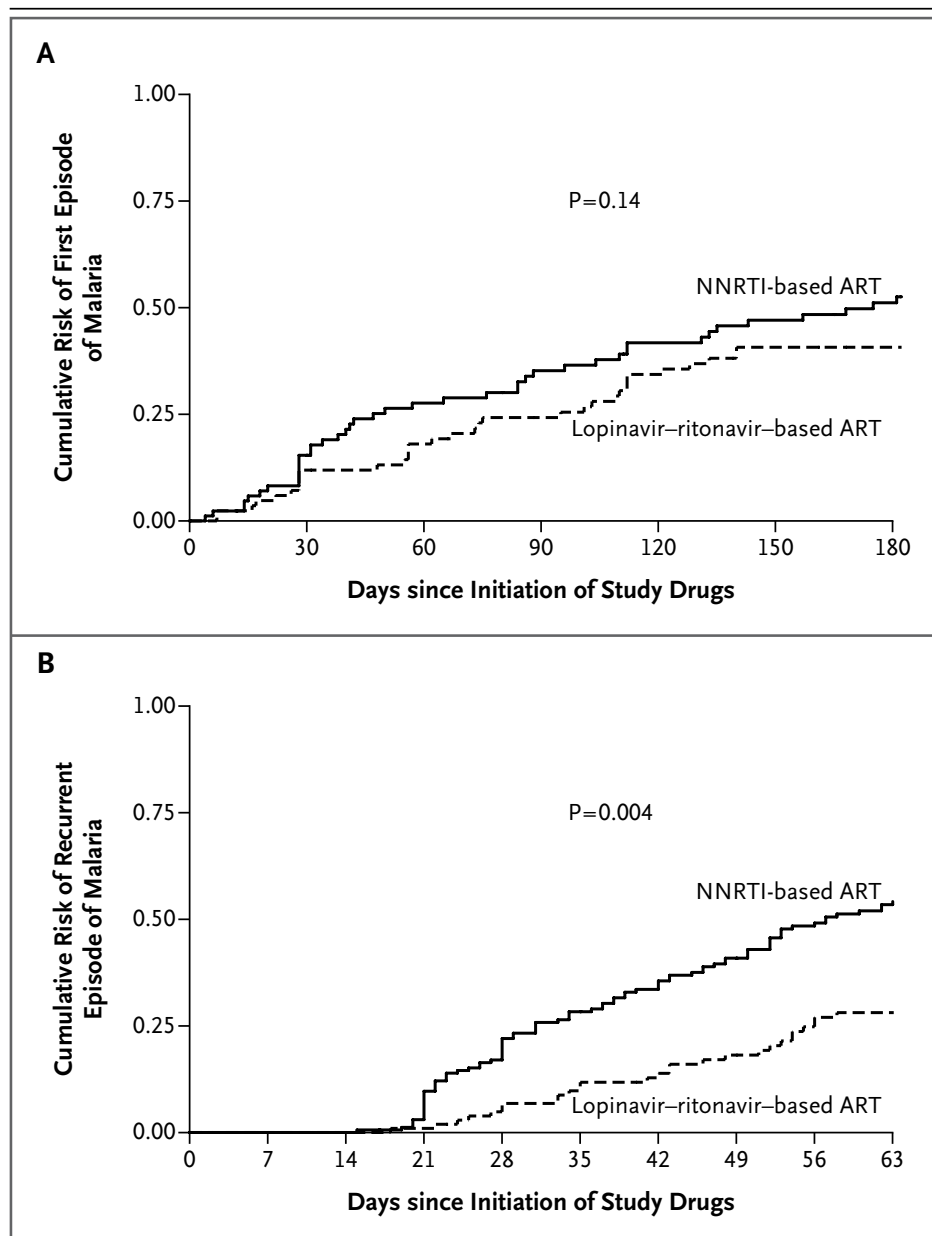

Figure 2. Selected Secondary Study Outcomes

Panel A shows Kaplan-Meier curves for the 6-month risk of a first episode of malaria, according to study group. Panel B shows Kaplan-Meier curves for the 63-day risk of recurrent malaria after treatment of malaria with artemetherlumefantrine, according to study group.

taking efavirenz (388 ng per milliliter [interquartile range, 164 to 563] vs. $97 \mathrm{ng}$ per milliliter [interquartile range, 61 to 124 ], $\mathrm{P}<0.001$ ). There was no significant association between the lumefantrine level on day 7 and the 63-day risk of recurrent malaria in the NNRTI group. In contrast, in the lopinavir-ritonavir group, children with lumefantrine levels of $300 \mathrm{ng}$ per milliliter or higher on day 7, as compared with children with lower drug levels, had a significantly reduced risk of recurrent malaria within 63 days (Fig. 3, and Table S3 in the Supplementary Appendix).

To determine whether there were direct antimalarial interactions between lopinavir and lumefantrine, two field isolates were cloned for in vitro drug-sensitivity testing. Modest synergy between lopinavir and lumefantrine was observed; data consistent with this are concave isobologram curves and mean fractional inhibitory concentrations near 0.5 (Fig. S1 in the Supplementary Appendix).

\section{DISCUSSION}

Among HIV-infected children in Uganda, a lopinavir-ritonavir-based antiretroviral regimen, as compared with an NNRTI-based regimen, reduced the incidence of malaria by $41 \%$. The primary benefit in the lopinavir-ritonavir group was conferred by a dramatic reduction in the risk of recurrent malaria after treatment with artemether-lumefantrine.

The protective effect of lopinavir-ritonavir against malaria could have resulted from direct antimalarial activity of lopinavir-ritonavir; inhibition of lumefantrine metabolism after treatment with artemether-lumefantrine, which would have extended the post-treatment prophylactic effect; or antimalarial synergy between lumefantrine and lopinavir. The possibility of direct antimalarial effects of HIV protease inhibitors is supported by in vitro studies showing activity of lopinavir at levels achievable in humans, ${ }^{3,5}$ in vivo studies showing activity against the rodent parasite $P$. chabaudi, ${ }^{5}$ activity against clinical isolates of P. falciparum and P. vivax, ${ }^{14}$ in vitro activity against $P$. falciparum in serum samples from patients receiving protease inhibitors, ${ }^{15}$ and action against $P$. falciparum cytoadherence and phagocytosis. ${ }^{16}$ In vitro antimalarial activity has not been reported with levels of NNRTIs that are attained with standard doses. ${ }^{2}$

The antimalarial activity of HIV protease inhibitors could theoretically be due to inhibition of plasmodial aspartic proteases that are biochemically similar to the HIV protease. ${ }^{3,5}$ However, we found only a nonsignificant trend toward a reduced risk of a first episode of malaria in the lopinavir-ritonavir group. Rather, protection against recurrent malaria accounted for most of the benefit of lopinavir-ritonavir, suggesting that the principal role of lopinavir-ritonavir with respect to protection against malaria is an effect on lumefantrine exposure. Children in the lopinavir-ritonavir group had significantly higher lumefantrine levels 7 days after the initiation of artemether-lumefantrine therapy than did chil- 
dren in the NNRTI group, and this effect was associated with a $59 \%$ reduction in the 63-day risk of recurrent malaria in the lopinavir-ritonavir group as compared with the NNRTI group. Increased lumefantrine exposure with concomitant lopinavir-ritonavir therapy, which was thought to be the result of inhibition of cytochrome P450 3A4 metabolism by ritonavir, was previously observed in healthy adults. ${ }^{6}$ In contrast, nevirapine and efavirenz both induce cytochrome P450 3A4 pathways, and the concurrent administration of either of these drugs with artemether-lumefantrine might diminish lumefantrine exposure. However, day 7 lumefantrine levels in the nevirapine group were similar to those reported previously in HIV-uninfected children receiving artemether-lumefantrine, ${ }^{17}$ suggesting that the greater protection in the lopinavir-ritonavir group than in the NNRTI group was due to inhibition of lumefantrine metabolism by lopinavir-ritonavir rather than to enhancement of cytochrome P450 3A4 metabolism by the NNRTIs.

The higher lumefantrine levels in the lopinavir-ritonavir group were not associated with a significantly increased risk of adverse events, with the exception of pruritus. Halofantrine, a related drug, is associated with prolongation of the QT interval and cardiac rhythm disturbances. In this study, no episodes of prolongation of the QTc interval were observed 3 days after the initiation of artemether-lumefantrine. ${ }^{18}$ We noted a trend toward a higher risk of serious adverse events after antimalarial therapy in the lopinavir-ritonavir group, with two episodes of neutropenia possibly related to the study drug. Given our limited statistical power for the comparison of uncommon events in this study and our limited evaluation of potential cardiotoxic effects, future studies of the safety of coadministration of lopinavir-ritonavir and lumefantrine are warranted. Increased lumefantrine exposure might also increase the selection of lumefantrine-resistant parasites. Genetic polymorphisms associated with decreased activity are selected by artemether-lumefantrine, ${ }^{19}$ suggesting that continued surveillance of the efficacy of artemether-lumefantrine therapy is warranted.

In vitro synergy in antimalarial activity between lopinavir and lumefantrine has been observed in laboratory strains of P. falciparum ${ }^{2}$ and in two isolates obtained from children in this

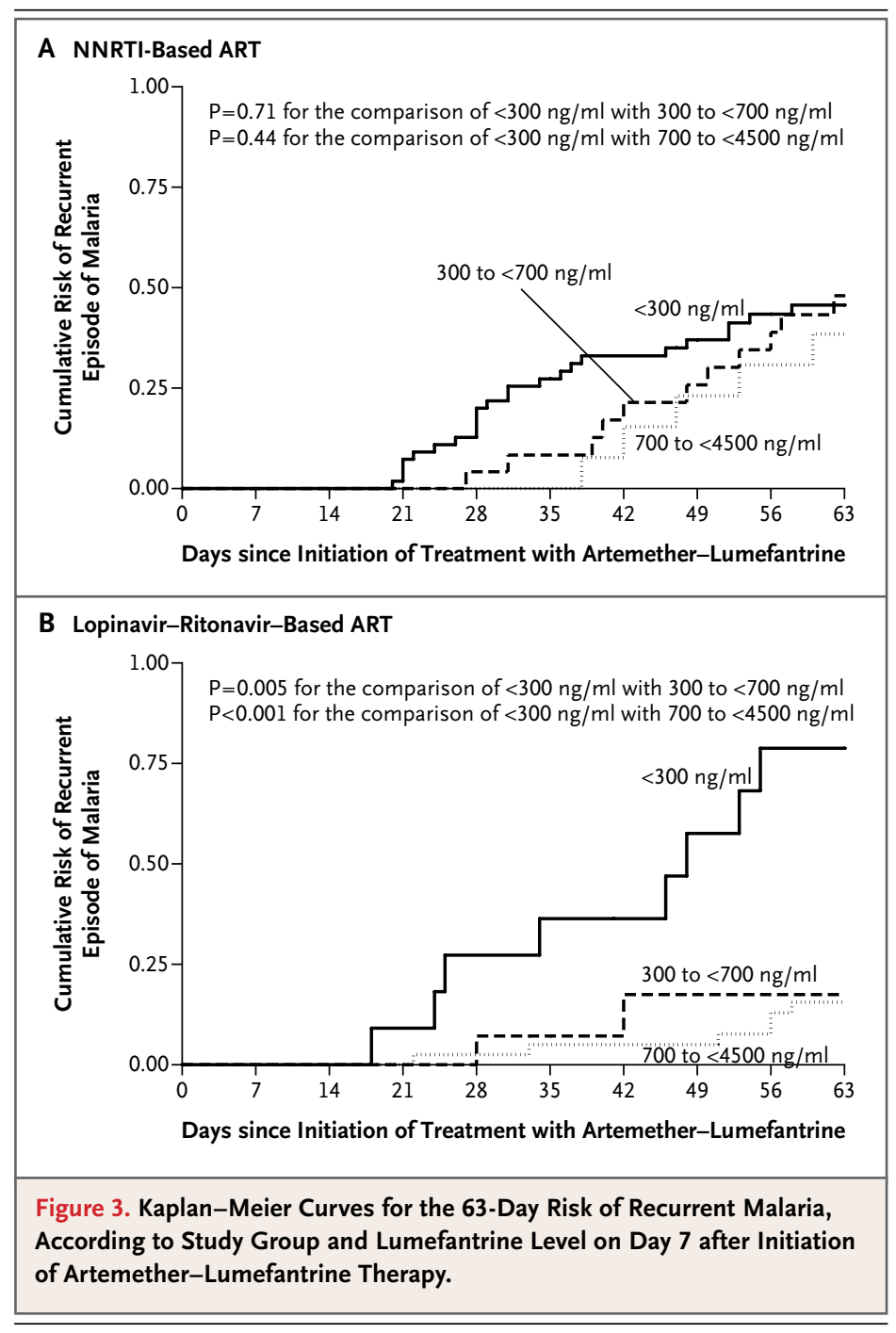

trial. Perhaps the synergistic antimalarial activity between lopinavir and lumefantrine enhanced the protective effect of prolonged lumefantrine exposure.

Our results suggest that a strategy of pharmacologic enhancement of exposure to antimalarial agents may be useful in reducing the burden of malaria, particularly in areas where the transmission intensity is high and recurrent malaria after treatment is commonplace. Although artemetherlumefantrine was very effective in clearing infections, the high risk of recurrent malaria after therapy, even with the use of insecticide-treated bed nets and trimethoprim-sulfamethoxazole prophylaxis, highlights the importance of posttreatment prophylaxis in high-transmission areas such as Uganda. In HIV-infected children requir- 
ing ART, it may be possible to use pharmacologic enhancement with lopinavir-ritonavir to provide protection from malaria. It is unlikely that an increase in the antimalarial dose without lopinavir-ritonavir therapy will achieve the same effect that we observed with lopinavir-ritonavir therapy in our cohort. The effects that are seen with increases in exposure of a drug may be several magnitudes greater than the effects that are possible with dose escalation - a finding that has been shown with respect to the enhancement of HIV protease inhibitors with ritonavir. ${ }^{20}$ In addition, lumefantrine exhibits saturable absorption, with the result that only modest increases in exposure are seen after dose escalation. ${ }^{21}$

In a randomized trial comparing lopinavirritonavir therapy with NNRTI therapy in children, virologic suppression rates were higher with lopinavir-ritonavir than with NNRTI - a finding that also supports the use of lopinavir-ritonavir. ${ }^{22,23}$ However, before changes in policy are implement- ed, the relative antiretroviral efficacies of various regimens should be studied further, the applicability of our findings to areas with a lower intensity of malaria transmission should be considered, and logistical challenges must be addressed. We are continuing to follow our cohort in order to evaluate long-term HIV efficacy outcomes. Previously, the high cost of the drugs and complex storage requirements represented considerable challenges in administering lopinavir-ritonavir in low-income countries. However, the declining cost of the drugs and the availability of a heat-stable lopinavir-ritonavir formulation suggest that these challenges are now surmountable. In conclusion, pharmacologic enhancement with the use of agents that alter drug metabolism may offer a means of improving the control of malaria.

Supported by a grant from the Eunice Kennedy Shriver National Institute of Child Health and Human Development.

Disclosure forms provided by the authors are available with the full text of this article at NEJM.org.

REFERENCES

1. Kamya MR, Gasasira AF, Achan J, et al. Effects of trimethoprim-sulfamethoxazole and insecticide-treated bednets on malaria among HIV-infected Ugandan children. AIDS 2007;21:2059-66.

2. Nsanzabana $C$, Rosenthal PJ. In vitro activity of antiretroviral drugs against Plasmodium falciparum. Antimicrob Agents Chemother 2011;55:5073-7.

3. Parikh S, Gut J, Istvan E, Goldberg DE, Havlir DV, Rosenthal PJ. Antimalarial activity of human immunodeficiency virus type 1 protease inhibitors. Antimicrob Agents Chemother 2005;49:2983-5.

4. Skinner-Adams TS, McCarthy JS, Gardiner DL, Hilton PM, Andrews KT. Antiretrovirals as antimalarial agents. J Infect Dis 2004;190:1998-2000.

5. Andrews KT, Fairlie DP, Madala PK, et al. Potencies of human immunodeficiency virus protease inhibitors in vitro against Plasmodium falciparum and in vivo against murine malaria. Antimicrob Agents Chemother 2006;50:639-48.

6. German P, Parikh S, Lawrence J, et al. Lopinavir/ritonavir affects pharmacokinetic exposure of artemether/lumefantrine in HIV-uninfected healthy volunteers. J Acquir Immune Defic Syndr 2009;51:424-9. 7. Okello PE, Van Bortel W, Byaruhanga $\mathrm{AM}$, et al. Variation in malaria transmission intensity in seven sites throughout Uganda. Am J Trop Med Hyg 2006;75:219-25.

8. Susceptibility of Plasmodium falciparum to antimalarial drugs: report on global monitoring: 1996-2004. Geneva: World Health Organization, 2005.

9. Table for grading the severity of adult and pediatric adverse events. Bethesda, MD: National Institute of Allergy and In- fectious Diseases, 2004 (http://www.niaid .nih.gov/LabsAndResources/resources/ DAIDSClinRsrch/Documents/ daidsaegradingtable.pdf).

10. Dorsey G, Staedke S, Clark TD, et al. Combination therapy for uncomplicated falciparum malaria in Ugandan children: a randomized trial. JAMA 2007;297:2210-9. 11. Huang L, Li X, Marzan F, Lizak PS, Aweeka FT. Determination of lumefantrine in small-volume human plasma by LC-MS/MS: using a deuterated lumefantrine to overcome matrix effect and ionization saturation. Bioanalysis 2012;4:157-66. 12. Nsobya SL, Kiggundu M, Nanyunja S, Joloba M, Greenhouse B, Rosenthal PJ. In vitro sensitivities of Plasmodium falciparum to different antimalarial drugs in Uganda. Antimicrob Agents Chemother 2010;54:1200-6.

13. François G, Hendrix L, Wery M. A highly efficient in vitro cloning procedure for asexual erythrocytic forms of the human malaria parasite Plasmodium falciparum. Ann Soc Belg Med Trop 1994;74:177-85.

14. Lek-Uthai U, Suwanarusk R, Ruengweerayut R, et al. Stronger activity of human immunodeficiency virus type 1 protease inhibitors against clinical isolates of Plasmodium vivax than against those of P. falciparum. Antimicrob Agents Chemother 2008;52:2435-41.

15. Redmond AM, Skinner-Adams T, Andrews KT, et al. Antimalarial activity of sera from subjects taking HIV protease inhibitors. AIDS 2007;21:763-5.

16. Nathoo S, Serghides L, Kain KC. Effect of HIV-1 antiretroviral drugs on cytoadherence and phagocytic clearance of Plasmodium falciparum-parasitised erythrocytes. Lancet 2003;362:1039-41. 17. Mwesigwa J, Parikh S, McGee B, et al. Pharmacokinetics of artemether-lumefantrine and artesunate-amodiaquine in children in Kampala, Uganda. Antimicrob Agents Chemother 2010;54:52-9.

18. van Vugt M, Ezzet F, Nosten F, et al. No evidence of cardiotoxicity during antimalarial treatment with artemether-lumefantrine. Am J Trop Med Hyg 1999;61:964-7. 19. Dokomajilar C, Nsobya SL, Greenhouse B, Rosenthal PJ, Dorsey G. Selection of Plasmodium falciparum pfmdr1 alleles following therapy with artemetherlumefantrine in an area of Uganda where malaria is highly endemic. Antimicrob Agents Chemother 2006;50:1893-5.

20. Palumbo P, Lindsey JC, Hughes MD, et al. Antiretroviral treatment for children with peripartum nevirapine exposure. N Engl J Med 2010;363:1510-20.

21. Palumbo PVA. NVP- vs LPV/r-based ART among HIV+ infants in resource limited settings: the IMPAACT1060 trial. Presented at the 18th Conference on Retroviruses and Opportunistic Infections, Boston, February 27-March 2, 2011. abstract.

22. Zeldin RK, Petruschke RA. Pharmacological and therapeutic properties of ritonavir-boosted protease inhibitor therapy in HIV-infected patients. J Antimicrob Chemother 2004;53:4-9.

23. Ashley EA, Stepniewska K, Lindegårdh $\mathrm{N}$, et al. Pharmacokinetic study of artemether-lumefantrine given once daily for the treatment of uncomplicated multidrug-resistant falciparum malaria. Trop Med Int Health 2007;12:201-8.

Copyright (c) 2012 Massachusetts Medical Society. 